\title{
VISUAL ACUITY FOLLOWING EXTRACAPSULAR CATARACT EXTRACTION IN DIABETES: A META-ANALYSIS
}

\author{
J. G. F. DOWLER, P. G. HYKIN, S. L. LIGHTMAN and A. M. HAMILTON \\ London
}

\begin{abstract}
SUMMARY
Although pre-operative retinopathy severity appears to be a major factor in determining the visual outcome of diabetic extracapsular cataract extraction, its precise relationship to post-operative visual acuity is ill defined. A meta-analysis was therefore carried out, and studies were included if pre-operative maculopathy and retinopathy status was sufficiently defined to permit discrimination of visual outcome between subgroups. Weighted mean proportions of eyes achieving a postoperative visual acuity $\geqslant 6 / 12$ were as follows: no retinopathy, $87 \%$; non-proliferative retinopathy with no maculopathy, $80 \%$; quiescent proliferative retinopathy with no maculopathy, 57\%; non-proliferative retinopathy with maculopathy, $41 \%$; quiescent proliferative retinopathy with maculopathy, $11 \%$; active proliferative retinopathy, 0 . Differences in visual outcome between groups were significant $\left(\chi^{2}=119.9\right.$, $p<0.0005)$, attributable mostly to the trend across groups $\left(\dot{\chi}^{2}\right.$ for trend $\left.=115.4, \quad p<0.0005\right)$. Logistic regression indicated that maculopathy was a more potent predictor of post-operative visual acuity $\leqslant 6 / 12$ (odds ratio 6.4, 95\% CI 4.13-9.94, $p<0.0005$ ) than quiescent proliferative retinopathy (odds ratio 3.33, 95\% CI $2.04-5.42, p<0.0005)$. The severity of retinopathy and maculopathy prior to cataract surgery in diabetics are the major determinants of post-operative visual acuity. Further study of the relationship between pre-operative retinopathy severity and the incidence of post-operative complications, progression of retinopathy and maculopathy is required to optimise the management of cataract in diabetes.
\end{abstract}

Cataract is the commonest cause of legal blindness in diabetics diagnosed after the age of 30 , and the second commonest cause after proliferative retinopathy in diabetics diagnosed before the age of $30 .^{1,2}$ In the United Kingdom $11 \%$ of cataract operations

Correspondence to: J. G. F. Dowler, FRCS, Moorfields Eye Hospital, City Road, London EC1V 2PD, UK. are carried out in diabetic patients, ${ }^{3}$ a figure equating to at least 10000 procedures per year. ${ }^{4}$ Such procedures in diabetics are associated with significant morbidity including post-operative progression of retinopathy, ${ }^{5-19}$ macular oedema, ${ }^{5-7,9-11,13-15,17,21}$ iris neovascularisation ${ }^{5,6,9,17,22-26}$ and fibrinous postoperative uveitis. ${ }^{7,9,27}$ As a reflection of this, postoperative visual acuity is frequently poorer than in non-diabetics. ${ }^{5-19,28-31}$ Several studies have examined the visual outcome of cataract surgery in diabetics, but populations were frequently small, and derived from specific categories of retinopathy. No study has been of sufficient size to encompass all retinopathy subgroups, and thus the precise relationship between pre-operative severity of retinopathy and post-operative visual acuity in diabetics undergoing cataract surgery is unclear. Meta-analysis, by synthesising the results of comparable studies, increases statistical power and permits an attempt to address this question using existing data.

\section{MATERIALS AND METHODS}

Studies of cataract extraction in diabetes were included in the analysis if the following criteria were satisfied: (1) Pre-operative retinopathy was sufficiently accurately defined to allow discrimination between eyes with no retinopathy, non-proliferative retinopathy, quiescent proliferative retinopathy and active proliferative retinopathy. (2) The presence or absence of maculopathy prior to surgery in eyes with retinopathy was specified; where pre-operative retinopathy severity was described as none, or minimal background, maculopathy was presumed to be absent. (3) The total number of eyes in each preoperative subgroup of retinopathy/maculopathy, and the numbers of eyes in each subgroup achieving a post-operative visual acuity $\geqslant 6 / 12$, were specified. (4) Extracapsular surgical technique was employed in the majority of cases, or the visual outcome of 
extracapsular cases was specified independently from the results of other forms of surgery.

Ten studies ${ }^{5-7,9,14,15,18,21,28,30}$ satisfied the criteria, of which seven were retrospective and three prospective. The results of 546 procedures were analysed. The commonest reason for exclusion was the absence of definite information regarding the presence or absence of maculopathy ${ }^{8,10,17}$ prior to surgery.

In each of the retinopathy groups, the weighted mean proportion of eyes with a post-operative vision $\geqslant 6 / 12$ was calculated. The $\chi^{2}$ test was used to identify differences between the rates in different retinopathy groups, and the $\chi^{2}$ for trend test was used to characterise the extent to which variation in rates between retinopathy groups could be attributed to an overall trend. Binary logistic regression was used to define the extent to which the presence of maculopathy or proliferative retinopathy affected the odds of a post-operative vision $\geqslant 6 / 12$. Techniques due to Fleiss $^{32}$ were employed to estimate the sample size in each retinopathy group which would allow discrimination between groups in a putative prospective trial with $95 \%$ confidence intervals and $80 \%$ statistical power.

\section{RESULTS}

A natural hierarchy of visual outcome across retinopathy groups was apparent, such that the more severe the pre-operative retinopathy, the lower the proportion of eyes achieving a postoperative visual acuity $\geqslant 6 / 12$ (Fig. 1 ). There was a significant difference between groups $\left(\chi^{2}=57.14\right.$, $p<0.0005)$, mostly attributable to a trend across groups $\left(\chi^{2}\right.$ for trend $\left.=54.5, p<0.0005\right)$. If the nonproliferative and quiescent proliferative retinopathy groups were further subdivided according to the presence or absence of maculopathy, the hierarchy was preserved, a lower proportion of eyes with

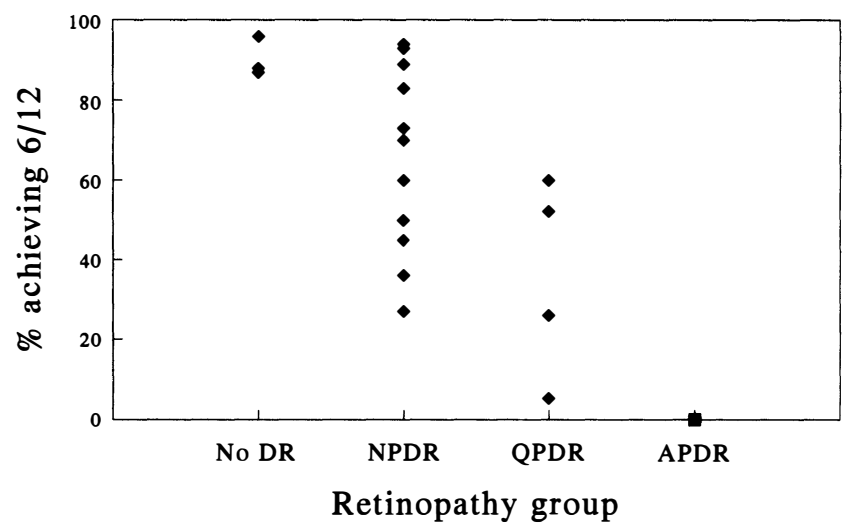

Fig. 1. Proportion of diabetic patients in different retinopathy groups achieving a post-operative visual acuity $\geqslant 6 / 12$. No DR, no diabetic retinopathy; NPDR, nonproliferative diabetic retinopathy; $Q P D R$, quiescent proliferative retinopathy; APDR, active proliferative retinopathy.

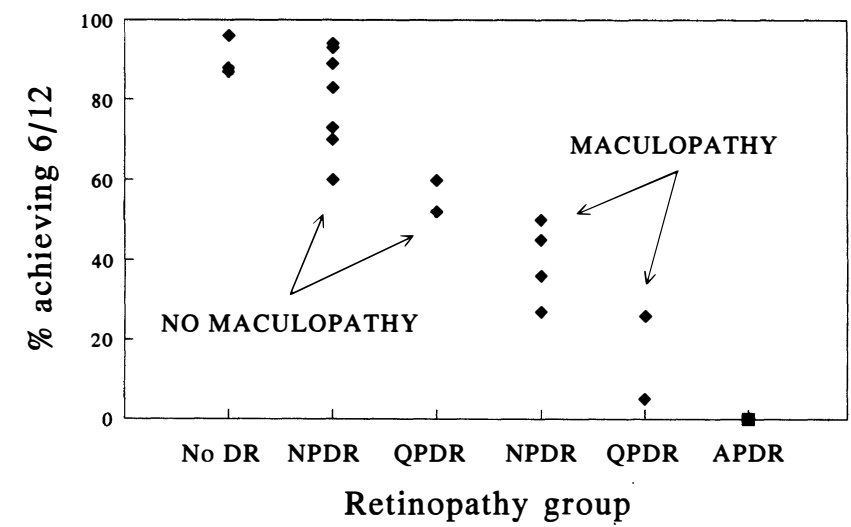

Fig. 2. Proportion of diabetic patients in different retinopathy groups achieving a post-operative visual acuity $\geqslant 6 / 12$ : effect of pre-operative maculopathy. No DR, no diabetic retinopathy; NPDR, non-proliferative diabetic retinopathy; $Q P D R$, quiescent proliferative retinopathy; $A P D R$, active proliferative retinopathy.

maculopathy achieving a post-operative visual acuity $\geqslant 6 / 12$ compared with eyes without maculopathy (Fig. 2). The difference between groups was significant $\left(\chi^{2}=119.9, p<0.0005\right)$, mostly attributable to a trend across the groups $\left(\chi^{2}\right.$ for trend $=$ $115.4, p<0.0005)$.

\section{$\% \geq 6 / 12 \quad$ Retinopathy Patients}

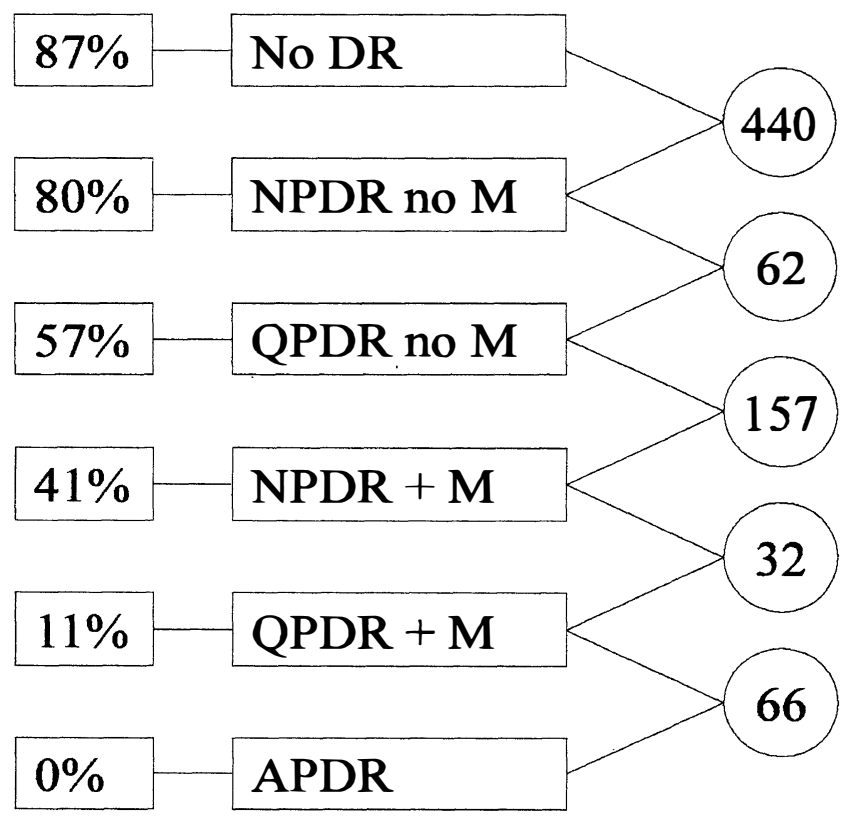

Fig. 3. Sample size estimations. $\% \geqslant 6 / 12$, weighted mean proportion of patients achieving post-operative visual acuity $\geqslant 6 / 12$ following diabetic extracapsular cataract extraction; retinopathy, pre-operative retinopathy severity; patients, patients required per arm of a putative prospective trial of $80 \%$ power and $95 \%$ confidence intervals. No DR, no diabetic retinopathy; NPDR, non-proliferative diabetic retinopathy; $Q P D R$, quiescent proliferative retinopathy; $A P D R$, active proliferative retinopathy; $+M$, maculopathy present before surgery, no $M$, no maculopathy present before surgery. 
Logistic regression indicated that maculopathy was a more potent predictor of visual acuity $\leqslant 6 / 12$ (odds ratio $6.4,95 \%$ CI $4.13-9.94, p<0.0005)$ than quiescent proliferative disease (odds ratio $3.33,95 \%$ CI $2.04-5.42, p<0.0005)$. The presence of active proliferative retinopathy was invariably associated with a post-operative visual acuity $\leqslant 6 / 12$.

The weighted mean percentages of patients achieving a post-operative visual acuity $\geqslant 6 / 12$ in different pre-operative retinopathy severity groups were used to calculate the sample sizes required in a putative prospective study to distinguish successively the following groups with statistical power of $80 \%$ and $95 \%$ confidence intervals (Fig. 3).

\section{DISCUSSION}

This meta-analysis details the proportion of diabetic eyes in each category of pre-operative retinopathy severity likely to achieve a post-operative visual acuity $\geqslant 6 / 12$ following extracapsular cataract extraction. This information is of value in advising patient of the likely visual outcome of surgery, in planning surgery, and in estimating sample size for a prospective trial. In addition, the results of this meta-analysis indicate that visual acuity following cataract surgery in diabetics is closely linked to the severity of retinopathy present before operation, and identifies maculopathy and proliferative retinopathy as particular risk factors for a poor visual result.

Maculopathy prior to surgery was associated with a sixfold increase in the risk of post-operative visual acuity $\leqslant 6 / 12$. This may be because cataract precludes recognition and timely and adequate treatment of maculopathy prior to surgery. Post-operative visual impairment may thus simply reflect the pre-operative status of the macula. However, several authors $^{5-7,9-11,13-15,17,21}$ have noted a tendency to deterioration of maculopathy following cataract surgery, which may take the form of a characteristic exudative retinopathy. ${ }^{10}$ This may occur even if a satisfactory response has been obtained to macular laser therapy prior to surgery. ${ }^{5}$ The cause of these changes is uncertain. The blood-aqueous barrier is impaired in diabetics, ${ }^{33}$ and this impairment is exacerbated by cataract surgery, ${ }^{34}$ being manifested clinically by an increased tendency to post-operative uveitis. ${ }^{27}$ Posterior diffusion of inflammatory mediators may further increase the permeability of the abnormal retinal microvasculature, either increasing the susceptibility to Irvine Gass syndrome ${ }^{21}$ or causing a deterioration of diabetic maculopathy..$^{10,11}$

The presence of active proliferative retinopathy prior to cataract surgery was invariably associated with a post-operative visual acuity $\leqslant 6 / 12$, and quiescent proliferative retinopathy was associated with a threefold increase in the risk of post-operative visual acuity $\leqslant 6 / 12$. The tendency to severe post- operative uveitis is particularly marked in patients with proliferative retinopathy, and fibrin membranes may form. ${ }^{7,9,27}$ Posterior dissemination of growth factors associated with the inflammatory response may be responsible for the tendency to postoperative progression of retinopathy and iris neovascularisation in such eyes. ${ }^{5-19,22-26}$ With deterioration of retinopathy, panretinal photocoagulation may be applied, and macular oedema may result. ${ }^{36}$ Alternatively, capillary non-perfusion may encroach upon the macula with consequent visual loss. Postoperative uveitis may be so severe as to impede photocoagulation where required, with further adverse effects of retinopathy. Finally, as noted above, inflammation may encourage deterioration of maculopathy. A variety of influences may therefore be responsible for the poor visual prognosis of cataract surgery in patients with proliferative retinopathy.

The studies included in this analysis are subject to some methodological variation. Definitions of retinopathy and maculopathy may have varied; in addition, there are inherent difficulties in grading retinopathy in eyes with cataract, and for this reason, in the metaanalysis, large retinopathy groupings were employed. Although surgery was extracapsular, some variation in technique is inevitable between centres. The indications for laser therapy are also likely to be variable, since some studies precede the publication of the findings of the Early Treatment Diabetic Retinopathy Study (ETDRS). Some discrepancy was apparent both within and between studies in the interval between surgery and visual acuity measurement, and because cataract surgery may be associated with progression of maculopathy and retinopathy, this may have affected the comparability of resuts. The majority of studies were retrospective, and the accumulation of study populations frequently took several years, so the quality of data obtained may be uneven. Finally, some studies acknowledged the presence of intercurrent pathology in a proportion of eyes under study, which may have affected visual results.

Additional bias may have resulted from the criteria for inclusion in the analysis. The commonest reason for exclusion was a failure to specify the numbers of patients with and without maculopathy achieving a post-operative visual acuity $\geqslant 6 / 12$. This resulted in the exclusion of several well-controlled studies. $^{8,10,17}$ However, visual outcome data are difficult to interpret without information regarding the pre-operative status of the macula, and we believe that the greater purity of the resulting sample justifies the exclusion of these studies.

Finally, bias inevitably results from the selection of cases included in studies submitted for publication (publication bias). ${ }^{37}$ One approach to elimination of 
such bias is to attempt the gathering of nonpublished data from the institutions at which the participating studies were conducted. This is, however, logistically impractical, and leaves uncorrected errors due to methodological anomalies. An alternative solution is to test the hypothesis generated by the meta-analysis in a large-scale prospective trial with defined inclusion criteria, surgical technique, indications for laser and review schedule. The authors are currently undertaking such a trial. Additional study is required to characterise the microvascular changes which occur at the macula following cataract surgery in diabetics, and to define their relationship to post-operative inflammation. The role of phacoemulsification, surface modification of intraocular lenses, and new intraocular lens materials has yet to be evaluated in this group of patients. Finally, the relationship between preoperative retinopathy severity and the incidence of post-operative complications, progression of retinopathy and maculopathy must be defined in order to optimise the management of cataract in diabetes.

Key words: Cataract, Diabetes, Meta-analysis

\section{REFERENCES}

1. Klein R, Klein BEK, Moss SE. Visual impairment in diabetes. Ophthalmology 1984;91:1-8.

2. Klein BEK, Klein R, Moss SE. Prevalence of cataracts in a population based study of persons with diabetes mellitus. Ophthalmology 1985;92:1191-6.

3. Harding JJ, Egerton M, van Heyningen R, Harding RS. Diabetes, glaucoma, sex and cataract: analysis of combined data from two case control studies. Br J Ophthalmol 1993;77:2-6.

4. Courtney P. The national cataract survey. I. Method and descriptive features. Eye 1992;6:487-92.

5. Benson WE, Brown GC, Tasman W, et al. Extracapsular cataract extraction with placement of a posterior chamber lens in patients with diabetic retinopathy. Ophthalmology 1993;100:730-8.

6. Cheng $\mathrm{H}$, Franklin SL. Treatment of cataracts in diabetics with and without retinopathy. Eye 1988; 2:607-14.

7. Cunliffe IA, Flanagan DW, George NDL, et al. Extracapsular cataract surgery in patients with lens implantation in patients with and without proliferative retinopathy. Br J Ophthalmol 1991;75:9-12.

8. Fung WE. Phacoemulsification and implantation of posterior chamber intraocular lens in eyes with quiescent proliferative diabetic retinopathy. Graefes Arch Clin Exp Ophthalmol 1987;225:251-3.

9. Hykin PG, Gregson RMC, Stevens JD, Hamilton AMP. Extracapsular cataract extraction in proliferative diabetic retinopathy. Ophthalmology 1993; 100:394-9.

10. Jaffe GJ, Burton TJ. Progression of nonproliferative diabetic retinopathy following cataract extraction. Arch Ophthalmol 1988;106:745-9.

11. Jaffe GJ, Burton TC, Kuhn E, et al. Progression of nonproliferative diabetic retinopathy and visual outcome after extracapsular cataract extraction and intraocular lens implantation. Am J Ophthalmol 1992; 114:448-56.
12. Levin ML, Kincaid MC, Eifler CW, Holt JE, et al. Effect of cataract surgery and intraocular lenses on diabetic retinopathy. J Cataract Refract Surg 1988; 14:642.

13. Pollack A, Dotan S, Oliver M. Course of diabetic retinopathy following cataract surgery. $\mathrm{Br} \mathrm{J}$ Ophthalmol 1991;75:2-8.

14. Pollack A, Dotan S, Oliver M. Progression of diabetic retinopathy after cataract extraction. Br J Ophthalmol 1991;75:547-51.

15. Pollack A, Leiba H, Bukelman A, Oliver M. Cystoid macular oedema following cataract extraction in patients with diabetes. Br J Ophthalmol 1992;76:221-4.

16. Pollack A, Leiba H, Bukelman A, et al. The course of diabetic retinopathy following cataract surgery in eyes previously treated by laser photocoagulation. $\mathrm{Br} \mathrm{J}$ Ophthalmol 1992;76:228-31.

17. Ruiz RS, Saatci OA. Posterior chamber lens implantation in eyes with inactive and active proliferative diabetic retinopathy. Am J Ophthalmol 1991; 111:158-62.

18. Sebestyen JG. Intraocular lenses and diabetes mellitus. Am J Ophthalmol 1986;101:425-8.

19. Sebestyen JG, Wafai MZ. Experience with intraocular lens implants in patients with diabetes. Am J Ophthalmol 1983;96:94.

20. Alpar JJ. Diabetes: cataract extraction and intraocular lenses. J Cataract Refract Surg 1987;13:43-6.

21. Menchini U, Bandello F, Brancato R, Camesasca FI, Galdini M. Cystoid macular oedema after extracapsular cataract extraction and intraocular lens implantation in diabetic patients without retinopathy. $\mathrm{Br} J$ Ophthalmol 1993;77:208-11.

22. Aiello LM, Wand M, Liang G. Neovascular glaucoma and vitreous haemorrhage following cataract surgery in patients with diabetes mellitus. Ophthalmology 1983;90:814-20.

23. Alpar JJ. Cataract extraction and diabetic retinopathy. Am Intraocul Implant Soc J 1984;10:433-7.

24. Beasley H. Rubeosis iridis in aphakic diabetics. JAMA 1970;213:128.

25. Prasad P, Setna PH, Dunne JA. Accelerated ocular neovascularisation in diabetics following posterior chamber lens implantation. Br J Ophthalmol 1990; 74:313-4.

26. Pavese T, Insler MS. Effects of extracapsular cataract extraction with posterior chamber lens implantation on the development of neovascular glaucoma in diabetics. J Cataract Refract Surg 1987;13:197-201.

27. Krupsky S, Zalish M, Oliver M, Pollack A. Anterior segment complications in diabetic patients following extracapsular cataract extraction and posterior chamber intraocular lens implantation. Ophthalmic Surg 1991;22:526-30.

28. Kennedy JE, Lim ASM, Ang BC. Posterior chamber intraocular lenses in diabetics. Aust NZ J Ophthalmol 1984;12:253-6.

29. Clayman HM, Jaffe NS, Light DS. Lens implantation and diabetes mellitus. Am J Ophthalmol 1979; 88:990-2.

30. Straatsma BR, Pettit TH, Wheeler N, Miyamasu W. Diabetes mellitus and intraocular lens implantation. Ophthalmology 1983;90:336-43.

31. Ngui MSH, Lim ASM, Chong AB. Posterior chamber intraocular lenses in diabetics: review of 63 patients. Int Ophthalmol 1985;8:257-9.

32. Fleiss JL. Statistical methods for rates and proportions. 2nd ed. New York: Wiley, 1981.

33. Oshika T, Kato S, Funatsu H. Quantitative assessment 
of aqueous flare intensity in diabetes. Graefes Arch Clin Exp Ophthalmol 1989;227:518-20.

34. Fergusson VMG, Spalton DJ. Continued breakdown of the blood-aqueous barrier following cataract surgery. Br J Ophthalmol 1992;76:453.

35. Moriarty AP, Spalton DL, Moriarty BJ, et al. Studies of the blood-aqueous barrier in diabetes mellitus. Am J Ophthalmol 1994;117:768-71.
36. Ferris FL, Podgor MJ, Davis MD. Diabetic Retinopathy Study Research Group. Macular edema in Diabetic Retinopathy Study patients. DRS report no. 12. Ophthalmology 1987;94:754-60.

37. Begg CB, Berlin JA. Publication bias: a problem in interpreting medical data. JR Statist Soc 1988; 151:419-63. 